\title{
Dieta estacional de guanacos (Lama guanicoe) y burros ferales (Equus asinus) en un ambiente semiárido de San Luis, Argentina
}

\author{
M. Laura Gomez Vinassa ${ }^{1, \bowtie}$ \& M. Beatríz Nuñez ${ }^{2}$ \\ ${ }^{1}$ Área de Ecología. Dto. de Bioquímica y Ciencias Biológicas. Facultad de Química, Bioquímica y Farmacia. Universidad \\ Nacional deSan Luis. San Luis, Argentina. ${ }^{2}$ Área de Biología. Dto. de Bioquímica y Ciencias Biológicas. Facultad deQuímica, \\ Bioquímica y Farmacia. Universidad Nacional de San Luis. San Luis, Argentina.
}

\begin{abstract}
Resumen. En la provincia de San Luis (Argentina), en un área ecotonal entre las regiones del Monte y del Chaco Seco, coexisten poblaciones de guanacos y burros asilvestrados. Dado que diferentes especies de herbívoros con masas corporales similares y viviendo en simpatría pueden estar sujetas a competencia interespecífica por alimento, se decidió determinar sus comportamientos alimentarios y analizar la existencia de solapamiento trófico. Se realizaron dos muestreos a lo largo de un año, en estación seca (ES) y húmeda (EH), para recolectar heces de cada especie y medir la disponibilidad relativa de alimento en el ambiente. Los hábitos alimentarios se evaluaron mediante análisis microhistológicos en términos de frecuencia relativa de aparición (FR\%) de las especies vegetales en las heces. La dieta del burro mostró variación estacional en el consumo de monocotiledóneas y se correlacionó de manera positiva con lo disponible en el ambiente durante la ES. Estuvo compuesta principalmente por pastos (75\% en ES y 50\% en EH), y en la época de lluvias aumentó el consumo de epífitas del género Tillandsia spp. (40\%). La dieta del guanaco también presentó variación estacional y se relacionó significativamente con la oferta ambiental durante la EH. Contuvo alrededor de $60 \%$ de leñosas, con un mayor porcentaje de epífitas y cactáceas en ES que en EH. Al comparar la composición total de las dietas, el porcentaje de similitud fue $20 \%$ en ES y $40 \%$ en EH, y se observó un valor elevado de similitud en relación al consumo de epífitas (74\%) y cactáceas (64\%) en EH. El resto del año, las dietas difirieron en composición y proporción de especies vegetales, por lo que se concluye que el solapamiento trófico entre burros y guanacos en el área de estudio es bajo.
\end{abstract}

[Palabras clave: simpatría, herbivoría, hábitos alimentarios, ungulados nativos, mamíferos exóticos]

\begin{abstract}
Aвstract. Seasonal diet of guanacos (Lama guanicoe) and feral donkeys (Equus asinus) in a semiarid environment of San Luis, Argentina. In the province of San Luis (Argentina), in an ecotonal area between Monte and Dry Chaco regions, populations of guanacos and feral donkeys coexist. Since sympatric herbivores with similar body masses may incur in interspecific competition for food, the food habits of guanacos and donkeys was determined to evaluate the degree of trophic overlap. Feces of each species were collected and relative food availability in the environment was measured in the dry (DS) and wet (WS) seasons. Relative frequency $(\% \mathrm{RF})$ of each vegetal item in scats was determined by microhistological analysis. According to our results, donkeys' diet showed seasonal variation in the consumption of monocots and was correlated with the available resources in the environment in DS. It was mainly composed of grasses (75\% in DS and 50\% in WS), with an increase in consumption of epiphytic plants in the genus Tillandsia spp. in the rainy season (40\%). Guanacos' diet also showed seasonal variation and was significantly related to environmental supply in WS. It contained about $60 \%$ of woody plants throughout the whole year, with a higher percentage of epiphytes and cactus in DS. The similarity in the total compositions of the diets was $20 \%$ in DS and $40 \%$ in WS, with higher values of similarity for epiphytes $(74 \%)$ and cactus $(64 \%)$ in WS. The rest of the year diets mostly differed in composition and proportion of plant items, so we conclude that the trophic overlap between these species in the study area is low.
\end{abstract}

[Keywords: sympatry, herbivory, food habits, native ungulates, exotic mammals]

\section{INTRODUCCIÓN}

La interacción entre especies nativas y exóticas en ambientes naturales es un tema clave y relevante para el manejo de fauna ya que al comprender sus mecanismos de coexistencia se obtienen herramientas indispensables para la conservación de la biodiversidad (Ramadori 2010; Villalobos and Zalba 2010). Para no en-

Editor asociado: Milesi Fernando

$\bowtie$ mlgomezvinassa@gmail.com trar en competencia, dos especies coexistentes con requerimientos ecológicos similares deben presentar una segregación en el uso de los recursos disponibles, en particular cuando se desarrollan en ambientes desérticos donde dichos recursos son escasos e impredecibles (Ovejero et al. 2011). Numerosos estudios han revelado el efecto negativo de los mamíferos 
exóticos sobre los nativos, en muchas ocasiones convirtiéndose en una amenaza para la biodiversidad en general (Aguirre Muñoz and Mendoza Alfaro 2009; Monge Meza 2009; Cuevas et al. 2010; Pereira Garbero et al. 2013). Investigaciones en regiones áridas de la Argentina han documentado los efectos de la competencia a partir del solapamiento trófico y el desplazamiento de las especies nativas hacia zonas menos productivas o muy degradadas (Bahamonde et al. 1986; Puig et al. 2001; Baldi et al. 2001, 2004; Borgnia et al. 2008).

En la región noroeste de la provincia de San Luis, Argentina, se conserva un área representativa del ecotono entre las regiones del Monte y del Chaco Seco argentino donde coexisten en la actualidad poblaciones de guanacos (Lama guanicoe Müller, 1776) y burros ferales (Equus asinus Linnaeus, 1758). Procedente de las zonas áridas de África, el burro es una especie que ha sido naturalizada con éxito en los desiertos americanos. En la actualidad se encuentra distribuida en todo el continente. A nivel mundial, muchos estudios demuestran los efectos adversos que las poblaciones de esta especie han generado en aquellas áreas donde han sido introducidos (Gordon and Illius 1989; Mishra et al. 2004; Hamrick et al. 2005; Australian Government 2011). En un ambiente natural, el mayor problema que generan es el ocasionado por competencia por alimento y agua con la fauna nativa, además de impactar fuertemente sobre las comunidades vegetales y la estructura del suelo (Álvarez Romero and Medelllín 2005; Beever and Aldridge 2011). Sin embargo, en nuestro país, las poblaciones de burros ferales han sido poco estudiadas, a pesar de que son consideradas invasoras y que habitan en numerosas áreas naturales destinadas a la conservación (Novillo and Ojeda 2008; Schüttler and Karez 2008). El guanaco es uno de los grandes herbívoros de Sudamérica. Si bien la especie se hallaba distribuida originalmente en diversos ambientes, en la actualidad se encuentra fragmentada en poblaciones pequeñas y relativamente aisladas. En la Argentina ocupa sólo 40\% de su distribución original (Tavarone 2004; Cinti 2005; APN 2006). Los principales factores vinculados con su declinación se relacionan con la alteración del hábitat, la competencia con especies introducidas y la caza indiscriminada (Puig et al. 1997; Amaya et al. 2001).

Por su tamaño y hábitos alimentarios, el guanaco y el burro han sido considerados equivalentes ecológicos (Novillo and Ojeda 2008). De acuerdo con esto y con lo establecido por Schoener (1974) y Baldi et al. (2004), quienes afirman que diferentes especies herbívoras con masas corporales similares y viviendo en simpatría pueden estar sujetas a competencia interespecífica por alimento, surgió la necesidad de evaluar la interacción trófica entre dichas especies. Los burros son herbívoros estrictos y suelen presentar una dieta compuesta exclusivamente por pastos. El guanaco, en cambio, ha sido descrito como un herbívoro generalista y mixto; su dieta varía a lo largo de su distribución geográfica (Iranzo et al. 2013) y es capaz de combinar hábitos de pastoreo con ramoneo según las condiciones del hábitat y de la oferta de alimentos (Puig et al. 1996, 1997, 2001; Muñoz and Simonetti 2013; Reus et al. 2014). Se espera, entonces, que en el área de estudio los guanacos se comporten como ramoneadores y que el grado de solapamiento dietario entre ambas especies sea bajo, de manera tal de favorecer la coexistencia. Se propusieron como objetivos: 1) analizar y comparar la composición estacional de las dietas del guanaco y del burro en una región semiárida en la provincia de San Luis, 2) estimar la disponibilidad relativa de alimento en el ambiente, y 3) evaluar la interacción trófica entre dichos herbívoros a través del análisis de su comportamiento alimentario.

\section{Materiales y Métodos}

\section{Área de estudio}

En la región noroeste de la provincia de San Luis (Argentina) se halla un ecotono formado por las Provincias Fitogeográficas del Chaco y Monte, pertenecientes al Dominio Chaqueño de la Región Neotropical (Anderson et al. 1970; Cabrera 1976). Allí se reconoce el bosque caducifolio xérico con un estrato de gramíneas, cactáceas y bromeliáceas terrestres mencionado por Morrone (2001) para la provincia del Chaco, como así también los matorrales abiertos con zigofiláceas de los géneros Larrea, Bulnesiay Plectrocarpamencionados por el mismo autor para la provincia del Monte.

El estudio se llevó a cabo dentro de los límites del Parque Nacional Sierra de las Quijadas (PNSQ, entre $32^{\circ} 20^{\prime}$ y $32^{\circ} 47^{\prime} \mathrm{S}$ y $67^{\circ} 10^{\prime}$ y $\left.66^{\circ} 58^{\prime} \mathrm{O}\right)$, un ambiente natural con escasa intervención humana y destinado a la conservación de la biodiversidad. El clima del área es árido serrano, típicamente continental, y se caracteriza por una amplitud térmica acentuada, tanto estacional como diaria. Los registros de temperatura tomadas en el área de 
estudio durante los últimos años muestran una máxima de $26.3^{\circ} \mathrm{C}$ y una mínima de $13.3^{\circ} \mathrm{C}$. Las precipitaciones son escasas (350 mm/año), distribuidas de manera irregular. Existen dos estaciones: la seca (ES, durante el invierno) y la húmeda $(\mathrm{EH}$, desde fines de primavera a principios de otoño) (APN 2006; Maero et al. 2007).

\section{Muestreos}

El trabajo de campo se desarrolló en un área de $\sim 100$ ha, donde la presencia conjunta de burros y guanacos fue confirmada por medio de su observación directa e indirecta a través de huellas, heces y rastros. Se realizaron dos campañas, una durante la estación seca en mayo de 2009 y otra durante la húmeda en enero de 2010. En cada período se recolectaron 15 alícuotas de heces de burro (encontradas con una separación de al menos 50 m) y 15 muestras de heces de guanaco, cada una con 15 pellets, extraídas de bosteaderos comunales diferentes. En todos los casos, las muestras fueron tomadas de defecaderos elegidos al azar que presentaron heces frescas, de manera tal de poder relacionarlos con los recursos disponibles en cada estación.

Para medir la disponibilidad relativa de alimento en el ambiente, en la misma área se trazaron cuatro transectas de $200 \mathrm{~m}$ de largo, con una separación de 500 m, y en cada una se dispusieron parcelas de $1 \mathrm{~m}^{2}$ cada $20 \mathrm{~m}$ (Sombra and Mangione 2005). Esto proporcionó un total de 40 parcelas, donde se registró la presencia de las especies vegetales allí presentes, de manera tal de calcular su frecuencia relativa de aparición (FR\%). Esta fue determinada para cada transecta, dividiendo el número de parcelas en los cuales una especie dada fue encontrada, por la suma total de las frecuencias de todas las especies identificadas (Puig et al. 1996).

\section{Colección de Referencias vegetales}

Para la identificación comparativa de los ítems vegetales presentes en las heces se confeccionó una colección de preparados histológicos semipermanentes, elaborados con epidermis foliares de las especies presentes en la zona de estudio. Dicha colección se encuentra disponible para su consulta o revisión en el Laboratorio de Ecología Nutricional del Área de Ecología de la Universidad Nacional de San Luis. Se utilizaron dos técnicas distintas de acuerdo a las características propias de cada hoja. Las monocotiledóneas fueron diafaniza- das: el material foliar se procesó en un molinillo eléctrico, luego fue hervido en alcohol etílico al 96\% durante 10 minutos y posteriormente en hidróxido de sodio otros 10 minutos más. La diafanización de los tejidos se realizó mediante la utilización de hipoclorito de sodio al 50\%, para luego colorearlo con safranina en solución alcohólica (Sombra and Mangione 2005). En el caso de las dicotiledóneas se utilizó la técnica de raspado, para lo cual las hojas fueron hidratadas mediante hervor en agua durante 10 minutos y luego se separó la epidermis con la ayuda de un pincel en el caso de hojas blandas, y de un bisturí, para láminas duras. El material obtenido fue aclarado con hipoclorito de sodio al $10 \%$ durante 5 minutos y luego coloreado con safranina alcohólica en los casos que fue necesario (Monge 1995). Todos los preparados fueron montados con gelatina glicerinada y fueron observados en microscopio (OLYMPUS CX31RT5F) a 100× y $400 \times$ para ser fotografiados (Cámara Digital Sony DSC-S650).

\section{Análisis microhistológico}

Para la preparación de las heces se siguieron los lineamientos generales del protocolo propuesto por Williams (1969): las fecas se secaron en estufa y se trituraron en un molinillo eléctrico. Se adicionaron $20 \mathrm{~mL}$ de una solución de alcohol en agua (70:30) a $200 \mathrm{mg}$ de polvo de heces y se dejó reposar durante 4 h. Posteriormente, se extrajo el sobrenadante y se agregó agua hirviendo. La solución se dejó en reposo por $12 \mathrm{~h}$. Por último, se aclararon con hipoclorito de sodio al 50\% para eliminar la clorofila, restos de mucus y los tejidos no epidérmicos. Las muestras se colorearon con safranina alcohólica y el medio de montaje utilizado fue la gelatina-glicerinada. Se confeccionaron tres preparados por muestra y se analizaron 40 campos microscópicos a 400× (Catán et al. 2003). A partir de cada muestra se calculó la frecuencia relativa de aparición $(\mathrm{FR} \%)$ de las especies vegetales en las heces $\left(\mathrm{FR} \%=\mathrm{N}^{\mathrm{o}}\right.$ de campos en los que el fragmento vegetal fue observado $\times 100 / \mathrm{N}^{\mathrm{o}}$ total de campos). Los ítems vegetales identificados en las dietas del guanaco y del burro fueron agrupados de manera convencional en cinco categorías: leñosas (árboles, arbustos y subarbustos), cactáceas, epífitas (especies del género Tillandsia), hierbas (especies herbáceas excepto gramíneas) y pastos (gramíneas). En algunos casos, la identificación llegó a nivel de especie, en otros sólo a género y en el caso de los cactus, a nivel de familia. 


\section{Análisis estadísticos}

Para comparar la composición estacional de las dietas del guanaco y el burro se aplicó la prueba no paramétrica de Wilcoxon (MannWhitney U) mediante el programa estadístico InfoStat (Di Rienzo et al. 2001). El solapamiento dietario se analizó a través del índice de Pianka (1973): $\mathrm{O}=\Sigma\left(\mathrm{P}_{\mathrm{ij}} \times \mathrm{P}_{\mathrm{ik}}\right) / \Sigma \mathrm{P}_{\mathrm{ij}}{ }^{2} \times \Sigma \mathrm{P}_{\mathrm{ij}}{ }^{2}$, donde $\mathrm{P}_{\mathrm{ij}}$ y $\mathrm{P}_{\mathrm{ik}}$ son las proporciones del recurso i utilizado por las especies j y k, respectivamente. La similitud de las dietas se estimó a partir del índice de Kulczynski (S, Olsen and Hansen 1977), que ofrece una medida directa de la proporción de ítems comunes consumidos por ambos herbívoros (Castellaro et al. 2004). Se calcula como: $\mathrm{S}=\left[\left(2 \mathrm{~W}_{\mathrm{i}}\right) /(\mathrm{a}+\mathrm{b})_{\mathrm{i}}\right] \times 100$, donde $\mathrm{W}_{\mathrm{i}}$ es el porcentaje menor de una determinada especie cuando se comparan sus porcentajes de consumo por dos animales diferentes y $(a+b)_{i}$ es la suma de estos dos porcentajes.

La preferencia por alguno de los grupos vegetales disponibles en el ambiente se calculó a través del índice de selectividad de Ivlev (1961): $E_{i}=\left(r_{i}-p_{i}\right) /\left(r_{i}+p_{i}\right)$ (Sombra and Mangione 2005), donde la Selectividad (E) está en función de la disponibilidad relativa de las categorías vegetales en el ambiente (p) y su uso relativo en la dieta (r). De acuerdo con Puig et al. (1996) se fijaron los valores -0.3 y 0.3 como límites para el "rango de indiferencia" (i.e., uso proporcional a la disponibilidad ambiental).

La diversidad de los ítems vegetales en la dieta y en el ambiente fue calculada a partir del índice estandarizado de Shannon-Wiener y sus diferencias fueron analizadas estadísticamente mediante la Prueba $t$ de Hutcheson (Puig et al. 1996, 1997; Moreno 2001; González and Briones-Salas 2012; Granados et al. 2014). Por último, mediante la prueba de correlación de Pearson se evaluó la correspondencia entre la FR\% de especies presentes en las heces de los animales respecto de la FR\% en el ambiente en ambas estaciones. En todos los análisis, las hipótesis nulas estadísticas fueron rechazadas cuando $P 0.05$ (i.e., los valores de los estadísticos fueron considerados significativos).

\section{Resultados}

\section{Disponibilidad de recursos alimentarios}

El área de estudio en el PNSQ exhibió una riqueza promedio de 47 especies vegetales. $\mathrm{El}$ género que se presentó con mayor frecuencia relativa promedio fue Tillandsia $(32.2 \%)$, segui- do por leñosas como Plectrocarpa (8.47\%), Ehretia(6.49\%)yCyclolepis(5.56\%).Pappophorum $(7.41 \%)$ fue el más frecuente entre los pastos y Tephrocactus ( $4.56 \%$ ) entre las cactáceas. La disponibilidad relativa de recursos alimentarios en el ambiente varió entre estaciones, observándose un aumento en la proporción de leñosas, epífitas y cactáceas en la estación húmeda y una mayor oferta de hierbas y pastos en la estación seca, quizás como consecuencia de las lluvias abundantes durante la estación húmeda previa a la de muestreo (Tabla 1). Los valores de diversidad obtenidos fueron de $\mathrm{H}^{\prime}=1.49$ en la período de sequía y de $\mathrm{H}^{\prime}=1.44$ en el húmedo, sin diferencias estadísticamente significativas entre sí $\left(t_{344}=1.13\right.$, $P>0.05)$.

\section{Dieta del guanaco y del burro}

De las 47 especies de plantas disponibles en promedio en el ambiente, $70 \%$ fueron encontradas en la dieta del guanaco y $65 \%$ en la de los burros. En la dieta del guanaco se identificaron 31 ítems vegetales (Tabla 1). Los géneros más consumidos, considerando su valor promedio entre estaciones, fueron Tillandsia (28.25\%) y Prosopis (26.71\%), seguidos por las cactáceas $(8.35 \%)$ y Cordobia argentea juntoconGomphrenacolosacanavar.andersonii (6.51\% y $6.39 \%$ respectivamente). En general el grupo de las leñosas fue el más representado, con una FR\% cercana a 50\% (Figura 1). No se detectaron diferencias significativas en la composición estacional de su dieta $(n=31, W=850$, $P=0.07)$. La diversidad de los ítems alimentarios consumidos por el guanaco fue mayor durante la estación húmeda $\left(\mathrm{H}^{\prime}=0.96\right)$ que durante la seca $\left(\mathrm{H}^{\prime}=0.78 ; t_{1300}=6.58, P<0.001\right)$.

En la dieta del burro se identificaron 34 ítems vegetales (Tabla 1). Los géneros más consumidos, considerando su valor promedio entre estaciones, fueron Pappophorum (24.32\%), Tillandsia (20.9\%) y Trichloris(10.67\%). En general el grupo de los pastos fue el más representado, encontrándose con una FR\% superior al 60\% (Figura 1). No se detectaron diferencias significativas en la composición estacional de su dieta $(\mathrm{n}=34 ; \mathrm{W}=1325 ; P=0.06)$. La diversidad de los ítems alimentarios consumidos por el burro fue mayor en la estación seca $\left(\mathrm{H}^{\prime}=1.19\right)$ que en la húmeda $\left(\mathrm{H}^{\prime}=0.85 ; t_{1080}=10.85, P<0.001\right)$.

\section{Similitud entre las dietas}

De los 44 ítems vegetales identificados en las heces de ambos herbívoros, 21 especies fueron comunes. La composición de las dietas 

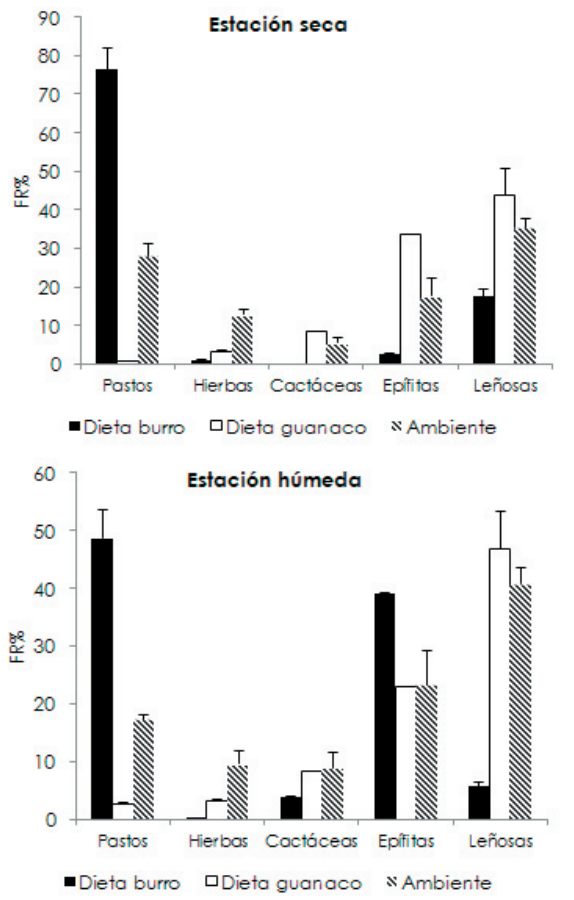

Figura 1. Frecuencia relativa porcentual promedio (+DE) de las categorías de plantas en las dietas del guanaco y del burro y en el ambiente en el Parque Nacional Sierra de las Quijadas en dos estaciones del año.

Figure 1. Mean $(+\mathrm{SD})$ relative percentage frequency of plant categories (grasses, forbs, cacti, epiphytes and woody plants) in guanacos (open) and feral donkeys (full) diets and available in the environment (dashed) of Parque Nacional Sierra de las Quijadas in the dry ("Estación seca") and wet ("Estación Húmeda") seasons. del guanaco y del burro fue diferente durante la estación seca ( $\mathrm{W}=1019 ; P=0.01)$ pero no durante la estación húmeda ( $\mathrm{W}=1277 ; P=0.68)$. De acuerdo al índice de Pianka, el grado de solapamiento total entre las dietas durante la estación seca fue bajo $(\mathrm{O}=0.21)$ y se duplicó hacia la estación húmeda $(\mathrm{O}=0.40)$. Resultados similares se obtuvieron con el índice de Kulczynski, que arrojó $18.5 \%$ de similitud en el periodo seco y $42 \%$ en el húmedo. Los valores más altos se presentaron en la estación húmeda en relación al consumo de epífitas $(73.84 \%)$ y de cactáceas $(63.73 \%)$. El grupo de las hierbas presentó cerca de $40 \%$ de similitud en las dietas en ambas estaciones. Respecto a la diversidad de los ítems presentes en la dieta de ambos herbívoros, se encontraron diferencias significativas en ambas estaciones (seca: $t_{1020}=14.68, P<0.001$; húmeda: $t_{1242}=3.54$, $P<0.001)$.

\section{Selección del alimento}

De acuerdo a los valores del índice de Ivlev (Figura 2), el burro mostró una marcada preferencia hacia el grupo de los pastos en ambas estaciones, mientras que el guanaco los rechazó. Las hierbas obtuvieron los valores más bajos del índice para ambos herbívoros en relación a los demás grupos, como así también las epífitas y cactáceas en la estación seca para el burro. Los valores obtenidos para el guanaco respecto a especies leñosas,

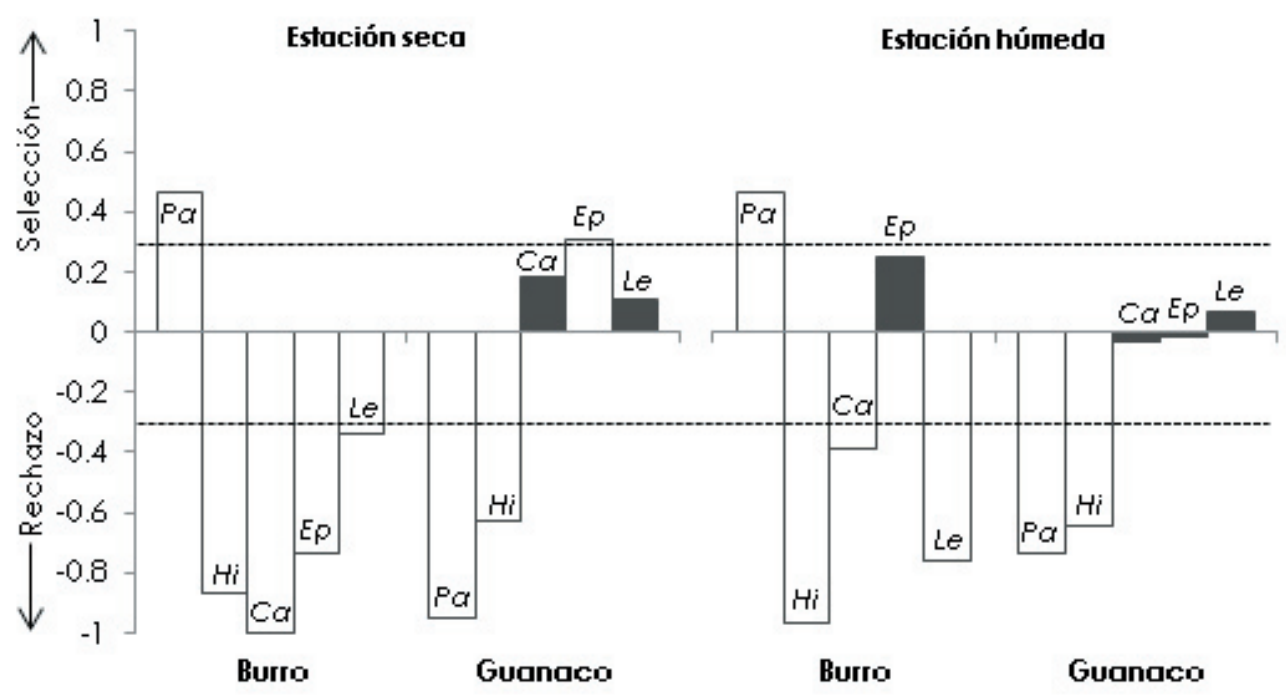

Figura 2. Preferencias dietarias del guanaco y del burro, de acuerdo al índice de selectividad de Ivlev. Se señalan en gris las categorías con valores del índice entre -0.3 y +0.3 , dentro del "rango de indiferencia" (sin selección). Categorías vegetales: $\mathrm{Pa}=$ pastos, $\mathrm{Hi}=$ hierbas, $\mathrm{Ca}=$ cactáceas, $\mathrm{Ep}=$ epífitas y Le=leñosas.

Figure 2. Dietary preferences of guanaco and burro according to electivity Index of Ivlev (positive values for positive selection). Grey bars indicate categories with index values between -0.3 and +0.3 , within the "indifference rank" (no selection). Plant categories: $\mathrm{Pa}=$ grasses, $\mathrm{Hi}=$ forbs, $\mathrm{Ca}=$ cactus, $\mathrm{Ep}=$ epiphytes, $\mathrm{Le}=$ shrubs and trees. 
Tabla 1. Frecuencia relativa porcentual promedio $( \pm \mathrm{DE})$ de especies vegetales en el área de estudio del Parque Nacional Sierra de las Quijadas y de ítems vegetales en las dietas del guanaco y del burro (FR\%ES=frecuencia relativa en la estación seca; FR\%EH=frecuencia relativa en la estación húmeda).

Table 1. Mean (+SD) relative percentage frequency of plant species in the diets of guanacos and feral donkeys in the study area of Parque Nacional Sierra de las Quijadas. (FR\%ES=relative frecuency in the dry season; FR\%EH=relative frecuency in the wet season).

\begin{tabular}{|c|c|c|c|c|c|c|}
\hline \multirow[t]{2}{*}{ Especie vegetal } & \multicolumn{3}{|c|}{ FR\% ES } & \multicolumn{3}{|c|}{$\mathrm{FR} \% \mathrm{EH}$} \\
\hline & Ambiente & Burro (n=15) & $\begin{array}{c}\text { Guanaco } \\
(\mathrm{n}=15)\end{array}$ & Ambiente & Burro $(n=15)$ & $\begin{array}{c}\text { Guanaco } \\
(\mathrm{n}=15)\end{array}$ \\
\hline PASTOS & $28.11(2.95)$ & $76.38(5.66)$ & $0.78(0.07)$ & $17.39(0.6)$ & $48.66(4.78)$ & $2.59(0.23)$ \\
\hline Pappophorum sp. & 0.52 & $5.21(5.32)$ & . & 1.2 & $7.11(4.47)$ & 0.13 \\
\hline P. caespitosum R.E.Fr. & $11.98(3.35)$ & $19.62(8.04)$ & $0.26(0.09)$ & . & $16.7(6.19)$ & $0.52(0.94)$ \\
\hline Pappophorum pappiferum (Lam.) & 0.52 & . & . & . & . & . \\
\hline $\begin{array}{l}\text { Kuntze } \\
\text { Pappophorum philippianum Parodi }\end{array}$ & . & . & . & 0.6 & . & . \\
\hline Trichloris sp. & . & $3.99(6.58)$ & . & 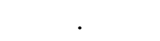 & $6.53(8.29)$ & 0.13 \\
\hline T. crinita (Lag.) Parodi & $1.56(1.09)$ & $5.21(5.43)$ & 0.13 & $2.99(1.08)$ & $5.08(6.25)$ & . \\
\hline T. pluriflora E. Fourn. f. pluriflora & . & 0.52 & . & . & . & . \\
\hline Chloris ciliata Sw. & . & $6.94(6.64)$ & 0.13 & 0.6 & $1.89(3.05)$ & 0.13 \\
\hline C. virgata Sw. & . & 0.17 & . & . & . & . \\
\hline Aristida sp. & . & $1.39(1.61)$ & . & . & $0.44(0.14)$ & . \\
\hline A. mendocina Phil. & . & $7.64(6.16)$ & . & . & $1.31(3.45)$ & $0.39(0.2)$ \\
\hline Panicum urvilleanum Kunth & $3.13(4.3)$ & $1.39(1.62)$ & . & $1.2(0.25)$ & $0.44(1.28)$ & . \\
\hline Setaria pampeana Parodi ex Nicora & $2.6(2.04)$ & 0.35 & . & . & $0.58(1.25)$ & . \\
\hline Sporobolus phleoides Hack. & $1.56(1.02)$ & $1.04(1.06)$ & . & 0.6 & $0.44(0.1)$ & . \\
\hline S. pyramidatus (Lam.) Hitchc. & $2.6(0.86)$ & $0.69(1.11)$ & . & $1.8(0.88)$ & . & . \\
\hline Neobouteloua lophostachya (Griseb.) & 0.52 & $0.69(1.15)$ & . & $\cdot$ & . & . \\
\hline Gould Digitaria californica (Benth.) & . & . & . & . & . & 0.13 \\
\hline $\begin{array}{l}\text { Henrard } \\
\text { Cottea pappophoroides Kunth }\end{array}$ & 0.52 & . & . & . & . & . \\
\hline G25 H & . & $3.3(4.08)$ & . & . & $0.73(1.1)$ & $0.39(0.2)$ \\
\hline G17 S & 0.52 & $0.87(1.08)$ & . & . & 0.15 & . \\
\hline Otras gramíneas no identificadas & $2.08(0.04)$ & $17.36(11.53)$ & $0.26(0.03)$ & 8.4 & $7.26(5.05)$ & $0.77(1.67)$ \\
\hline HIERBAS & $13(1.1)$ & $0.86(0.36)$ & $3.03(0.71)$ & $9.59(2.33)$ & 0.15 & $3.23(0.32)$ \\
\hline Portulaca sp. & . & 0.17 & . & . & . & . \\
\hline Sp20 H & . & . & $0.53(0.08)$ & . & 0.15 & $0.64(0.16)$ \\
\hline Sp1 H & . & . & 0.26 & 0.6 & . & $0.39(0.64)$ \\
\hline Glandularia sp. & $2.6(1.89)$ & . & $1.85(2.77)$ & . & . & $1.16(4.28)$ \\
\hline Sp23 H & . & . & . & . & . & 0.26 \\
\hline Solanum tweedianum Hook. & $1.04(0.02)$ & $0.69(2.08)$ & 0.13 & . & . & $0.39(0.17)$ \\
\hline Sp27 & $3.64(1.71)$ & . & 0.26 & $5.99(3.28)$ & . & $0.39(0.09)$ \\
\hline Otras hierbas no identificadas & $5.72(4.52)$ & . & . & $3(2.54)$ & . & . \\
\hline CACTÁCEAS & $5.73(1.09)$ & . & 8.32 & $8.99(2.56)$ & 3.92 & 8.38 \\
\hline Tephrocactus sp. & $3.13(3.13)$ & $?$ & $?$ & $5.99(4.04)$ & $?$ & $?$ \\
\hline Tephrocactus articulatus (Pfeiff) & $1.56(0.98)$ & $?$ & $?$ & 0.6 & $?$ & $?$ \\
\hline $\begin{array}{l}\text { Backeb var. articulatus } \\
\text { Opuntia sp. }\end{array}$ & $1.04(0.02)$ & $?$ & $?$ & $1.8(0.02)$ & $?$ & $?$ \\
\hline Cereus aethiops Haw & . & $?$ & $?$ & 0.6 & $?$ & $?$ \\
\hline Cactus & . & . & $8.32(6.21)$ & & $3.92(5.87)$ & $8.38(5.71)$ \\
\hline EPÍFITAS & $17.71(4.77)$ & 2.6 & 33.55 & $23.35(5.9)$ & 39.19 & 22.94 \\
\hline Tillandsia myosura Griseb. ex Baker & $1.04(0.02)$ & $?$ & $?$ & 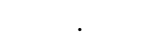 & $?$ & $?$ \\
\hline T. capillaris Ruiz and Pav. & $11.46(2.28)$ & $?$ & $?$ & $14.37(2.96)$ & $?$ & $?$ \\
\hline T. tricholepis Baker & $3.13(1.01)$ & $?$ & $?$ & $2.99(1.89)$ & $?$ & $?$ \\
\hline T. xiphioides Ker Gawl var. & $2.08(0.94)$ & $?$ & $?$ & $5.99(2.84)$ & $?$ & $?$ \\
\hline $\begin{array}{l}\text { Xiphioides } \\
\text { Tfllandsia spp. }\end{array}$ & & $2.6(2.74)$ & $33.55(12.18)$ & & $39.19(16.78)$ & $22.94(14.76)$ \\
\hline LEÑOSAS & $35.41(2.16)$ & $17.52(1.72)$ & $43.85(6.96)$ & $40.74(2.89)$ & $5.68(0.85)$ & $46.92(6.25)$ \\
\hline $\begin{array}{l}\text { Cercidium praecox (Ruiz, and Pav. } \\
\text { Ex Hook.)Harms }\end{array}$ & . & . & . & & 0.58 & 0.13 \\
\hline
\end{tabular}


Tabla 1. Continuación

Table 1. Continuation

\begin{tabular}{|c|c|c|c|c|c|c|}
\hline \multirow[t]{2}{*}{ Especie vegetal } & \multicolumn{3}{|c|}{ FR\% ES } & \multicolumn{3}{|c|}{$\mathrm{FR} \% \mathrm{EH}$} \\
\hline & Ambiente & Burro $(n=15)$ & $\begin{array}{c}\text { Guanaco } \\
(\mathrm{n}=15)\end{array}$ & Ambiente & Burro $(n=15)$ & $\begin{array}{c}\text { Guanaco } \\
(\mathrm{n}=15)\end{array}$ \\
\hline Lycium sp. & $3.13(4.28)$ & $2.08(3.11)$ & 0.4 & & . & $1.16(1.56)$ \\
\hline Atriplex sp. & $4.69(1.63)$ & $0.69(0.16)$ & $0.4(0.94)$ & $3.59(1.05)$ & 0.44 & $0.77(1.91)$ \\
\hline $\begin{array}{l}\text { Gomphrena colosacana Hunz. and } \\
\text { Subils var. andersonii Subils and }\end{array}$ & 0.52 & $4.69(9.07)$ & $0.92(1.55)$ & 0.6 & 0.15 & $11.86(10.17)$ \\
\hline $\begin{array}{l}\text { Hunz. } \\
\text { Prosopis sp. }\end{array}$ & 0.52 & $0.87(1.64)$ & $21.66(6.62)$ & . & $0.29(0.09)$ & $18.43(7.37)$ \\
\hline P. flexuosa D.C. & . & $0.69(0.94)$ & $7.13(5.67)$ & . & $2.32(1.93)$ & $6.19(5.01)$ \\
\hline P. torquata (Cav. ex Lag.) D.C. & 1.04 & . & . & $1.8(0.88)$ & . & . \\
\hline P. strombulifera (Lam.) Benth var. & . & . & . & $1.8(0.23)$ & . & . \\
\hline $\begin{array}{l}\text { Strombulifera } \\
\text { Ehretia cortesia Gottschling }\end{array}$ & $5.21(2.17)$ & . & . & $7.78(2.42)$ & . & . \\
\hline Suaeda divaricata Moq & 0.52 & . & . & $1.8(1.19)$ & 0.15 & . \\
\hline Sp3 H & . & $4.51(4.95)$ & $1.59(4.39)$ & 1.2 & 0.29 & $2.71(3.47)$ \\
\hline Cordobia argentea (Griseb.) Nied. & 1.04 & $3.47(5.23)$ & $10.96(5.4)$ & . & $1.02(1.61)$ & $2.06(1.34)$ \\
\hline Sp9 S & 0.52 & $0.35(0.05)$ & $0.53(1.05)$ & . & $0.29(0.08)$ & $1.55(1.31)$ \\
\hline Plectrocarpa tetracantha Gillies ex & $6.77(3.23)$ & . & . & $10.18(2.57)$ & . & . \\
\hline Henna aphylla (Cav.) H.S. Irwin, & . & . & . & & . & $0.9(1.75)$ \\
\hline $\begin{array}{l}\text { and Barneby } \\
\text { Larrea cuneifolia Cav. }\end{array}$ & $2.08(2.1)$ & . & . & $2.4(2.35)$ & . & . \\
\hline Acacia aroma Gillies ex Hook. and & . & 0.17 & . & . & . & . \\
\hline Arn Geoffraea decorticans (Gillies ex & 0.52 & . & 0.13 & 0.6 & 0.15 & 0.26 \\
\hline $\begin{array}{l}\text { Hook. and Arn.) Burkart } \\
\text { Capparis atamisquea Kuntze }\end{array}$ & 0.52 & . & 0.13 & . & . & . \\
\hline Zuccagnia punctata Cav. & $1.04(0.02)$ & . & . & 1.2 & . & 0.77 \\
\hline Gochnatia glutinosa (D. Don) & 0.52 & . & . & - & . & 0.13 \\
\hline $\begin{array}{l}\text { Hook. and Arn. } \\
\text { Monttea aphylla (Miers) Benth and }\end{array}$ & . & . & . & $1.2(0.03)$ & . & $\cdot$ \\
\hline $\begin{array}{l}\text { Hook } \\
\text { Cyclolepis genistoides D. Don }\end{array}$ & $5.73(1.68)$ & . & . & $5.39(0.91)$ & . & . \\
\hline Ximena americana $\mathrm{L}$. & . & . & . & 0.6 & . & . \\
\hline Ephedra triandra Tul. Emend. J.H. & 1.04 & . & . & . & . & . \\
\hline $\begin{array}{l}\text { Hunz. } \\
\text { Mimozyganthus carinatus (Griseb.) }\end{array}$ & . & . & . & 0.6 & . & . \\
\hline $\begin{array}{l}\text { Burkart } \\
\text { Otras dicotiledóneas no } \\
\text { identificadas }\end{array}$ & . & $2.6(2.13)$ & $10.44(9.65)$ & . & $2.47(1.44)$ & $15.98(7.63)$ \\
\hline
\end{tabular}

epífitas y cactáceas se mantuvieron dentro del "rango de indiferencia" lo que equivale a un consumo proporcional a la disponibilidad ambiental. Algo similar se observó en los resultados obtenidos en el análisis de correlación entre los ítems presentes en el ambiente y en la dieta del guanaco (estación húmeda: $\mathrm{r}_{8}=0.94$, $P=0.02$; estación seca: $\mathrm{r}_{8}=0.50, P=0.39$; anual: $\left.\mathrm{r}_{8}=0.78, P=0.12\right)$. La correspondencia entre las especies vegetales en la dieta del burro y en el ambiente fue algo mayor, pero no estadísticamente diferente en el periodo seco $\left(\mathrm{r}_{8}=0.90\right.$, $P=0.07)$ que en el húmedo $\left(\mathrm{r}_{8}=0.06, P=0.93\right.$; anual $r_{8}=0.30, P=0.62$ ).

\section{Discusión}

En Argentina existen numerosos estudios acerca de los hábitos alimentarios del guanaco y de su interacción trófica con otros herbívoros domésticos como ovejas, cabras y vacas (Candia and Dalmasso 1995; Puig et al. 1996, 1997, 2001; Baldi et al. 2004; Guiñazú 2007; Muñoz and Simonetti 2013; Soler et al. 2013). Sin embargo, la dieta del burro, y específicamente las relaciones tróficas entre ambas especies, han sido poco estudiadas (Borgnia et al. 2008; Reus et al. 2014), a pesar de que habitan simpátricamente en varias regiones de nuestro país y se estima que podrían presentar competencia por los recursos disponibles.

Según Borgnia et al. (2008) la dieta del burro en la región del Altiplano argentino está compuesta principalmente por pastos, al igual que lo observado por Reus et al. (2014) para la región del Monte en San Juan. Esto coincide con nuestros resultados donde las gramíneas aparecieron con un porcentaje anual superior a $60 \%$. La especie más consumida por el burro 
enambasestacionesfuePappophorumcaespitosum, que también se presentó con una elevada FR\% en el ambiente. Cabe destacar que ésta es considerada una especie forrajera natural de gran importancia para la alimentación del ganado doméstico en regiones semiáridas, debido particularmente a su componente hoja y alto contenido de proteína bruta (PB 7\%) (Ávila et al. 2008). Durante la estación seca el burro aumentó el consumo de especies leñosas, lo cual podría relacionarse con la elevada concentración de nitrógeno que éstas poseen y su mayor contenido de agua (Quiroga et al. 2008). Mediante el ramoneo de árboles y arbustos los animales obtienen mayores niveles de proteína y energía en la dieta, y compensan la calidad baja que les ofrece el pastizal durante el período seco (Rossi et al. 2008).

La dieta del guanaco, en cambio, se destacó por incluir una variedad amplia de grupos vegetales. Las especies leñosas fueron las que se encontraron en mayor proporción en su dieta a lo largo de todo el año. En el Parque Provincial Ischigualasto, San Juan, se observaron resultados similares: los arbustos fueron el principal aporte a su dieta, seguidos de pastos, bromeliáceas y cactus (Reus et al. 2014). En nuestro sitio de estudio, la especie leñosa más consumida fue Prosopis sp., considerada una especie de ramoneo de buena calidad forrajera debido a su porcentaje de proteína (PB 12\%) y relativamente bajo contenido de polifenoles (Luquez de Mucciarelli et al. 1982; Rossi et al. 2008; Puig et al. 2009). El guanaco presenta diversas dietas a lo largo de su distribución en Argentina. Si bien distintos autores sostienen que los pastos son la base y el componente más importante en la alimentación del guanaco, seguidas por los arbustos y las hierbas (Candia and Dalmasso 1995; Puig et al. 1996, 1997; Fraser 1999; Puig et al. 2001), en nuestro trabajo los pastos presentaron valores muy bajos de FR\% en su dieta. En primer lugar, esa diferencia podría deberse a que la FR\% de especies leñosas en el ambiente es mayor a la de los pastos a lo largo de todo el año y en segundo lugar, podría deberse al consumo elevado de estos últimos por parte del burro. Es importante mencionar las diferencias anatómicas y fisiológicas entre ambos herbívoros, donde el funcionamiento diferencial de sus aparatos digestivos (pseudo-rumiante en el guanaco y fermentador en el caso del burro) les permite adoptar distintas estrategias de forrajeo, lo cual resulta en una separación de su nicho trófico (Gordon and Illius 1989; Menard et al. 2002). De esta manera, los equinos pueden sostener tasas altas de consumo de forraje de baja calidad, sobre todo en época de sequía, cuando los pastos escasean, mientras que el guanaco puede ramonear de forma eficiente sobre una oferta más amplia de arbustos y subarbustos. Todo esto favorecería la coexistencia de ambas especies en la región.

Durante la estación seca, la dieta del guanaco incluyó una notable cantidad de Tillandsiaspp. Resultados similares fueron encontrados en el área del Parque Provincial Ischigualasto en San Juan (Guiñazú 2007), donde dicho ítem representó más del $25 \%$ de la composición botánica de la dieta del herbívoro. Respecto a este género Es importante destacar que tanto el burro en la estación húmeda como el guanaco en la seca poseen un consumo elevado de esta especie, que aparece con un alto porcentaje en sus dietas. En cuanto a composición química, es un género de plantas que contienen gran acumulación de sodio y alrededor de $75 \%$ de agua. Además, estudios de la calidad nutricional de estas epífitas en zonas áridas y semiáridas indican que su contenido en proteína varía entre $5.7 \%$ y $6.6 \%$, y que pueden ser utilizadas por los rumiantes durante todo el año, en particular durante la época de sequía, cuando el forraje es escaso (Beltrán et al. 2009).

El conocimiento de la selectividad alimentaria es de gran interés en el manejo de fauna. A partir de nuestros resultados observamos una alta selección del burro hacia el grupo de los pastos, mientras el guanaco incorporó en su dieta la misma proporción de ítems que se encontraron disponibles, poniendo de manifiesto su comportamiento generalista. De acuerdo con varios autores (Puig et al. 1996; Baldi et al. 2004; Muñoz and Simonetti 2013) la dieta del guanaco es flexible y puede variar según la estación del año, el área de forrajeo o ante la presencia de herbívoros domésticos. Esta plasticidad le permite superar situaciones de escasez en la oferta alimentaria y reducir la competencia con el ganado. En nuestra área de estudio se observó una superposición de la distribución espacial de las especies y un porcentaje total de solapamiento dietario inferior al 50\%, situación que podría variar en función a la densidad de animales en el ambiente y su dinámica a lo largo del tiempo. Algo similar fue señalado en el noroeste de Argentina, donde se investigó el uso de hábitat y la interacción entre ungulados nativos y domésticos, y se determinó que las vicuñas pueden mantener sus preferencias de forrajeo y coexistir con herbívoros domésticos cuando éstos se encuentran a densidades bajas o medias; sin embargo, al aumentar su densidad, se produce 
una segregación espacial y las vicuñas tienden a utilizar ambientes subóptimos (Arzamendia and Vilá 2014).

La razón fundamental de nuestro trabajo fue obtener información que pudiera servir de sustento a planes de manejo y conservación, no sólo de una especie emblemática como es el guanaco sino también de la biodiversidad regional asociada. Actualmente, las autoridades administrativas y técnicas del PNSQ sostienen que la población de burros asilvestrados contribuye a la degradación de los valores de conservación de tipo arqueológico, paleontológico y de biodiversidad; sin embargo, a pesar de estar identificada como un problema, aún no se han tomado acciones de manejo por carecer de un diagnóstico adecuado de su efecto general (Agudelo Henríquez 2012). Un factor a tener en cuenta para continuar evaluando su impacto en el ambiente es la elevada proporción de pastos que los burros asilvestrados consumen. Cuando hay sobrepastoreo, la selección que hacen los animales de ciertas especies vegetales en relación a otras provoca a largo plazo cambios en la cobertura vegetal y en la densidad de plantas (Pelliza Sbriller et al. 2002). Esto puede afectar a los herbívoros nativos que de ellas dependen. En áreas naturales de Monte, la presencia del burro podría estar alterando la disponibilidad de alimento para otras especies que consumen principalmente pastos, como la mara o liebre patagónica (Dolichotis patagonum Zimmermann, 1780), especie Vulnerable de acuerdo a su categoría nacional de conservación (Ojeda et al. 2012). Consideramos necesario complementar este estudio con evaluaciones a largo plazo de las especies introducidas, abordando un número mayor de variables como uso de hábitat, comportamiento, densidad y dinámica poblacionales, y disponibilidad de recursos limitantes. De esta manera se obtendrá una visión más integral de su interacción y sus efectos sobre el ambiente.

AgRAdECIMIENTOS. Queremos agradecer al personal del PNSQ por el apoyo técnico y logístico en el campo, a A. Mangione por sus aportes y por brindarnos a través de su proyecto de investigación el marco necesario para el desarrollo de este trabajo. A M. Lugo, C. Carossio y G. Molina por su colaboración en la identificación de especies vegetales. A E. Menoyo, A.C. Ochoa, A. Gatica y N. Denkiewicz por sus aportes valiosos a este manuscrito, y a todos aquellos que colaboraron en las campañas de muestreo.

\section{REFERENCIAS}

Agudelo Henríquez, W. J. 2012. Evaluación preliminar de la ocupación del burro silvestre Equus asinus en dos sectores del Parque Nacional Sierra de las Quijadas, San Luis, Argentina. Tesis de Maestría en Manejo de Vida Silvestre. Universidad Nacional de Córdoba.

Aguirre Muñoz, A., and R. Mendoza Alfaro. 2009. Especies exóticas invasoras: impactos sobre las poblaciones de flora y fauna, los procesos ecológicos y la economía en Capital natural de México, vol. II: Estado de conservación y tendencias de cambio. Conabio, México.

Álvarez Romero, J., and R. Medellín. 2005. Equus asinus. Vertebrados superiores exóticos en México: diversidad, distribución y efectos potenciales. Instituto de Ecología, Universidad Nacional Autónoma de México. Bases de datos SNIB-CONABIO. Proyecto U020. México. D.F.

Amaya, J., J. Von Thüngen, and D. De Lamo. 2001. Relevamiento y distribución de guanacos en la Patagonia. Comunicación Técnica N 107. Área RRNN Fauna. INTA EEA Bariloche. INTA-GTZ-TöB.

Anderson, D., J. Del Águila, and A. Bernardon. 1970. Las formaciones vegetales en la Provincia de San Luis. Investigaciones Agropecuarias, INTA, Buenos Aires, Argentina. Serie 2, Biología y Producción Vegetal, Vol. VII N³.

Administración de Parques Nacionales (APN). 2006. Plan de Manejo Parque Nacional Sierra de las Quijadas. Delegación Regional Centro.

Arzamendia, Y., and B. Vilá. 2014. Vicugna habitat use and interactions with domestic ungulates in Jujuy, Northwest Argentina. Mammalia 79(3):267-278.

Australian Government. 2011. Feral Horse (Equus caballus) and Feral Donkey (Equus asinus). Department of Sustainability, Environment, Water, Population and Communities.

Ávila, R., E. Quiroga, C. Ferrando, and L. Blanco. 2008. Dinámica de la calidad a lo largo del año de dos gramíneas nativas de Los Llanos de La Rioja. Sitio Argentino de Producción Animal. EEA INTA, La Rioja.

Bahamonde, N., S. Martin, and A. Pellizer Sbriller. 1986. Diet of guanaco and red deer in Neuquén Province, Argentina. J Range Manage 39:22-24.

Baldi, R., S. Albon, and D. Elston. 2001. Guanacos and sheep: evidence for continuing competition in arid Patagonia. Oecologia 129:561-570.

Baldi, R., A. Pelliza-Sbriller, D. Elston, and S. Albon. 2004. High potencial for competition between guanacos and sheep in Patagonia. Journal of Wildlife Management 68(4):924-938.

Beever, E. A., and C. L. Aldridge. 2011. Influences of free-roaming equids on sagebrush ecosystems, with a focus on Greater Sage-Grouse. Pp. 273-290 in: S. T. Knick and J. W. Connelly (eds.). Greater Sage-Grouse: ecology and 
conservation of a landscape species and its habitats. Studies in Avian Biology (vol. 38), University of California Press, Berkeley, CA.

Beltrán, L., V. Gámez, O. Loredo, V. Bañuelos, and D. Rincón. 2009. Análisis químico proximal de Tillandsia recurvata L. para evaluar su potencial como planta forrajera. VI Simposio Internacional de Pastizales.

Borgnia, M., B. Vilá, and M. Cassini. 2008. Interaction between wild camelids and livestock in an Andean semi-desert. Journal of Arid Environments 72:2150-2158.

Cabrera, A. 1976. Regiones fitogeográficas de la República Argentina. En L. R. Parodi (ed.), Enciclopedia Argentina de Agricultura y Jardinería, ed. 2:2-85. Acme S.A.C.I., Buenos Aires.

Candia, R., and A. Dalmasso. 1995. Dieta del guanaco (Lama guanicoe) y productividad del pastizal en la Reserva La Payunia, Mendoza (Argentina). Multequina Nro. 4.

Castellaro, G., T. Ullrich, B. Wackwitz, and A. Raggi. 2004. Composición botánica de la dieta de alpacas (Lama pacos L.) y llamas (Lama glama L.) en dos estaciones del año, en praderas altiplánicas de un sector de la Provincia de Parinacota, Chile. Agricultura Técnica 64(4).

Catán, A., C. Degano, and L. Larcher. 2003. Modificaciones a la técnica micro histológica de Peña Neira para especies forrajeras del Chaco Semiárido Argentino. Quebracho 10:71-75.

Cinti, R. 2005. Fauna Argentina: dramas y prodigios del bicherío. 1ra edición. Buenos Aires: Emecé Editores. ISBN 950-04-2657-9.

Cuevas, M., A. Novillo, C. Campos, M. Dacar, and R. Ojeda. 2010. Food habits and impact of rooting behaviour of the invasive wild boar, Sus scrofa, in a protected area of the Monte Desert, Argentina. Journal of Arid Environments 74: 1582-1585.

Di Rienzo, J., C. W. Robledo, F. Casanoves, M. G. Balzarini, L. A. González, et al. 2001. InfoStat. Estadística y biometría. Facultad de Ciencias Agropecuarias. Universidad Nacional de Córdoba. Versión beta. infostat@agro.uncor.edu.

Fraser, M. 1999. A comparison of the diet composition of guanacos (Lama guanicoe) and sheep when grazing swards with different clover: grass ratios. Small Ruminant Research. Elsevier Science 32:231-241.

González, G., and M. Briones- Salas. 2012. Dieta de Odocoileus virginianus (Artiodactyla: Cervidae) en un bosque templado del norte de Oaxaca, México. Rev Biol Trop 60(1):447-457.

Gordon, I. J., and A. W. Illius. 1989. Resources partitioning by the ungulates on the Isle of Rhum. Oecologia 79:383389.

Granados, D., L. Tarango, G. Olmos, J. Palacio, F. Clemente, and G. Mendoza. 2014. Dieta y disponibilidad de forraje del venado cola blanca Odocoileus virginianus thomasi (Artiodactyla: Cervidae) en un campo experimental de Campeche, México. Rev Biol Trop 62(2):699-710.

Guiñazú, V. 2007. Uso de recursos alimentarios por Lama guanicoe en el Parque Provincial Ischigualasto (San Juan, Argentina). Tesina de Licenciatura en Ciencias Naturales. Ciclo de Licenciaturas "San Pedro Nolasco", Universidad del Aconcagua.

Hamrick, R., T. Pirgalioglu, S. Gunduz, and J. Carroll. 2005. Feral Donkey Equus asinus populations on the Karpaz peninsula, Cypru. Eur J Wildlife Res 51:108-116.

Iranzo, E. C., J. Traba, P. Acebes, B. A. González, C. Mata, et al. 2013. Niche Segregation between Wild and Domestic Herbivores in Chilean Patagonia. Plos One 8(3):e59326. doi:10.1371/journal.pone.0059326.

Ivlev, V. S. 1961. Experimental ecology of the feeding of fishes. Yale University Press. New Haven.

Luquez De Mucciarelli, S., J. Cid, M. Molins DE Pedernera, M. Lucas De Orellano, and C. Guardia. 1982. Composición química y valor nutritivo de dos especies de Prosopis (P. caldenia y P. torquata). A B A 46(1-2):1-10.

Maero, I. S., D. Rivarola, and G. Tognelli. 2007. Cartografía morfodinámica para el Parque Nacional Sierra de las Quijadas. San Luis. Argentina. Gestión Ambiental 14:57-77

Menard, C., P. Duncan, G. Fleurance, J. Y. Georges, and M. Lila. 2002. Comparative foraging and nutrition of horses and cattle in European wetlands. Journal of Applied Ecology 39:120-133.

Mishra, C., S. Wieren, P. Ketner, I. Heitkönig, and H. H. Prins. 2004. Competition between livestock and wild bhral Pseudois nayaur in the Indian Tras-Himalaya. Journal of Applied Ecology 41:344-354.

Monge, S. 1995. Características epidérmicas de dicotiledóneas encontradas en las dietas de herbívoros en la Reserva de la Biósfera de Ñacuñán (Santa Rosa, Mendoza). Multequina 4.

Monge Meza, J. 2009. Efecto de mamíferos exóticos en Costa Rica. Biocenosis 22(1-2).

Moreno, C. E. 2001. Métodos para medir la biodiversidad. M\&T-Manuales y Tesis SEA, vol. 1. Zaragoza. Pp. 84.

Morrone, J. J. 2001. Biogeografía de América Latina y el Caribe. M\&T-Manuales \& Tesis. SEA, vol. 3. Zaragoza. Pp. 148.

Muñoz, A. E, and J. A. Simonetti. 2013. Diet of guanaco in sheep-free rangeland in Tierra del Fuego, Chile. Ciencias e Investigación Agraria. Animal Ecology 40(1):185-191.

Novillo, A., and R. A. Ojeda. 2008. The exotic mammals of Argentina. Biological Invasion 10(8):1333-1344.

Ojeda, R. A., V. Chillo, and G. B. Díaz Isenrath (eds.). 2012. Libro Rojo de Mamíferos Amenazados de la Argentina. Sociedad Argentina para el Estudio de los Mamíferos (SAREM). ISBN en trámite.

Olsen, F., and R. Hansen. 1977. Food relations of free-ranging horses to livestock and big game, Red Desert, Wyoming. Journal of Range Management 30:17-20.

Ovejero, J. R., P. Acebes, J. E. Malo, J. Traba, M. E. Mosca Torres, et al. 2011. Lack of feral livestock interference with native guanaco during the dry season in a South American desert. Eur J Wildlife Res 57:1007-1015. 
Pelliza Sbriller, A., G. Bonvissuto, and N. Bonino. 2002. Selección de dieta por herbívoros en Patagonia: Escalas Espaciales y Temporales. Comunicación Técnica N47. Instituto Nacional de Tecnología Agropecuaria.

Pereira Garbero, R., J. M. Barreneche, G. Laufer, F. Achaval, and M. Arim. 2013. Mamíferos invasores en Uruguay, historia, perspectivas y consecuencias. Rev Chil Hist Nat 86:403-421.

Pianka, E. R. 1973. The structure of lizard communities. Annual Review of Ecology and Systematics 4:53-74.

Puig, S., F. Videla, S. Monge, and V. Roig. 1996. Seasonal variations in guanaco diet (Lama guanicoe, Muller 1776) and food availability in Northern Patagonia, Argentina. Journal of Arid Environments 34:215-224.

Puig, S., F. Videla, and M. Cona. 1997. Diet and abundance of the guanaco (Lama guanicoe, Müller 1776) in four habitats of northern Patagonia, Argentina. Journal of Arid Environments 36:343-357.

Puig, S., F. Videla, M. Cona, and S. Monge. 2001. Use of food availability by guanacos (Lama guanicoe) and livestock in Northern Patagonia (Mendoza, Argentina). Journal of Arid Environments 47:291-308.

Puig, S., M. Cona, F. Videla, and E. Mendez. 2009. Diet of the mara (Dolichotis patagonum), food availability and effects of an extended drought in Northern Patagonia (Mendoza, Argentina). Mammalian Biology 75:389-398.

Quiroga, E., R. Ávila, C. Ferrando, and L. Blanco. 2008. Dinámica de la calidad y de material verde en los componentes morfológicos de dos gramíneas nativas del Chaco Árido. Revista Argentina de Producción Animal 28(1):349-543.

Ramadori, D. 2010. Erradicación de Especies Exóticas Invasoras. Dirección de Fauna Silvestre. Secretaría de Ambiente y Desarrollo Sustentable de la Nación.

Reus, M. L., B. Peco, C. De Los Ríos, S. M. Giannoni, and C. M. Campos. 2013. Trophic interactions between two medium-sized mammals: the case of the native Dolichotis patagonum and the exotic Lepus europaeus in a hyper-arid ecosystem. Acta Theriol 58:205-214.

Reus, M. L., F. M. Cappa, N. Andino, V. E. Campos, C. de los Ríos, and C. M. Campos. 2014. Trophic interactions between the native guanaco (Lama guanicoe) and the exotic donkey (Equus asinus) in the hyper-arid Monte desert (Ischigualasto Park, Argentina). Studies on Neotropical Fauna and Environment 49(3):159-168.

Rossi, C., A. Pereyra, G. González, M. De León, and P. Chagra Dib. 2008. Composición química, contenido de polifenoles totales y valor nutritivo en especies de ramoneo del sistema silvopastoril del Chaco árido argentino. Zootecnia Tropical 26(2).

Schoener, T. W. 1974. Competition and the form of habitat shift. Theoretical Population Biology 6:265-307.

Schüttler, E., and C. Karez (eds.). 2008. Especies exóticas invasoras en las Reservas de Biosfera de América Latina y el Caribe. Un informe técnico para fomentar el intercambio de experiencias entre las Reservas de Biosfera y promover el manejo efectivo de las invasiones biológicas. UNESCO, Montevideo.

Soler, R. M., G. M. Pastur, M. V. Lencinas, and L. Borrelli. 2013. Seasonal diet of Lama guanicoe (Camelidae: Artiodactyla) in a heterogeneous landscape of South Patagonia. Bosque 34(2):129-141.

Sombra, M., and A. Mangione. 2005. Obsessed with grasses? The case of mara Dolichotis patagonum (Caviidae: Rodentia). Rev Chil Hist Nat 78:401-408.

Tavarone, E. 2004. Análisis de la factibilidad de reintroducción del guanaco (Lama guanicoe) en el Parque Nacional Quebrada del Condorito. Tesis de Maestría. Programa de Postgrado en Manejo de Vida Silvestre. Universidad Nacional de Córdoba.

Villalobos, A., and S. Zalba. 2010. Continuous feral horse grazing and grazing exclusion in mountain pampean grasslands in Argentina. Acta Oecologica 36:514-519.

Williams, O. 1969. An improved technique for identification of plant fragments in herbivore feces. Journal of Range Management 22:51-52. 